\title{
Research on the Vocal Music Teaching Model Based on Computer Platform
}

\author{
Youjun Dai \\ Lanzhou Vocational Technical College, Gansu, Lanzhou, 730000
}

Keywords: Vocal music teaching platform; data mining; vocal training

\begin{abstract}
Computer technology is proposed to apply in vocal music teaching in view of the problems in traditional vocal music teaching. First, vocal music teaching needs are analyzed which shows that vocal music teaching mainly solves music characterization and teaching platform functions and data transmission problems. Then teaching platform is established which includes training platform, user information platform, performance platform and data mining platform.
\end{abstract}

\section{Introduction}

Vocal music is an important skill in music learning and an important part of music education ${ }^{[1]}$. With the national investment in education, music education has become an important part of national quality education. In traditional vocal music teaching, students' voice condition is basically judged by teachers through listening while students can't know their own status, which would influence their vocal music learning. Sound digitization provides sound quantitative analysis for vocal music teaching with the development of computer technology ${ }^{[2-6]}$. This paper discusses the application of computer platform in vocal music teaching.

\section{Demand analysis of vocal music teaching model}

\subsection{Music representation of computer}

The computer program is composed of binary code, with which the music information can only be processed by encoding into binary information. The flow of processing music is shown in figure 1.

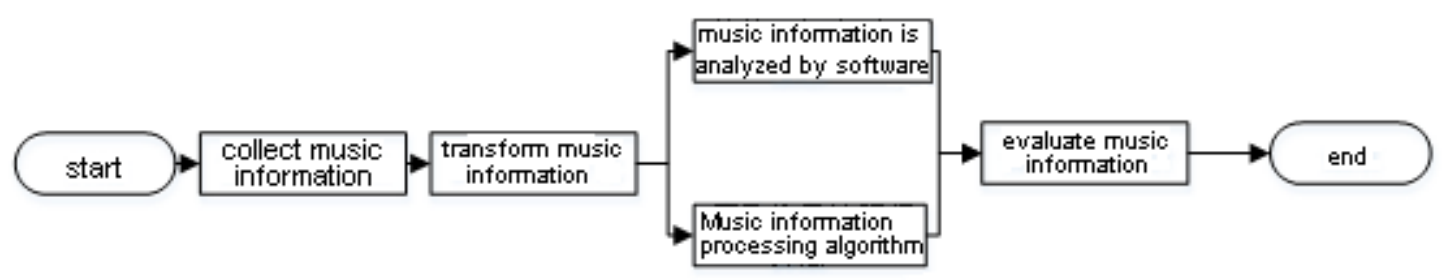

Figure 1 music information processing flow

Music information processing includes collection, transformation, processing and evaluation of information. Students' voice information can be collected by equipment such as microphone. Music information transformation is to transform sound information into digital signals. Sound information can be analysed through software such as CakewallProAudio and Samplitude or tone matching algorithm. Music information evaluation is to evaluate students' voice.

\subsection{Demand analysis of vocal music teaching platform}

There are various content in examination of vocal music teaching, such as students' vocal music learning status, after-class learning status, interaction with teachers, student performance management, and student information management

The students' learning status of vocal music mainly inspects students' intonation, rhythm and so on. The process used to rely on teachers to observe students' learning by listening to their vocalizations, while computer technology is used to investigate students' vocal learning. Students only need to upload their own vocal music information to the system, which adopts the vocal music 
information processing algorithm to evaluate vocal music, and students can query their own learning results in the system. It takes a lot of time to learn vocal music, so students need to practice after class. How do students evaluate their practice needs to be solved. In the process of music learning, students might encounter problems more or less, and they can ask teachers' help through the system. Teachers can provide guidance according to students' questions. Figure 2 shows the function of vocal music teaching platform.

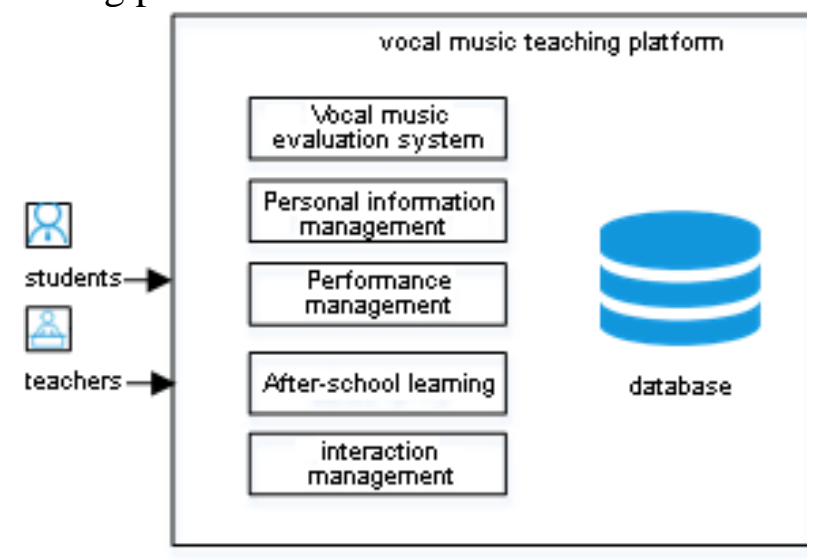

Figure 2 Function diagram of vocal music teaching platform

The personal information in the platform mainly includes students' major, specialty and vocal learning status. Performance management is used to store students' performance and help them analyze problems in practice. In addition, teachers can analyze the advantages and disadvantages of students' vocal music learning through students' academic performance, so that students can better practice vocal music.

\subsection{Analysis of vocal music teaching platform data demand}

Sound information is the core component of vocal music teaching. This transmission is difficult because the voice message is often loud. Multi-thread technology is used to transmit sound information for better transmission of sound information platform.

\section{Content of vocal music teaching platform construction}

\subsection{Platform construction framework}

The core function of vocal music platform is virtual vocal music platform. The platform includes: rhythm training, sound training, virtual ensemble, digital audio, etc. Figure 3 shows the construction framework of the vocal virtual platform.

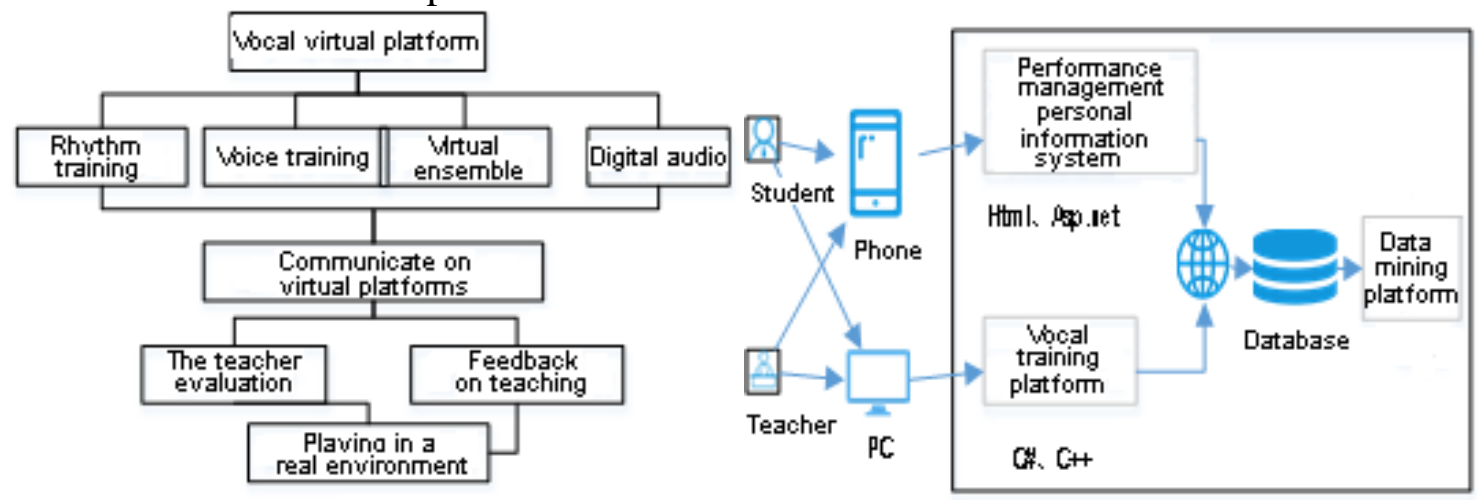

Figure 3. construction framework of vocal virtual platform

Virtual vocal music platform is the key platform of vocal music teaching. It provides a platform for students to practice vocal music. The external platform also includes user management and performance management. 


\subsection{Core technologies for platform construction}

In the construction of vocal music teaching platform, due to the need to upload students' vocal music information and at the same time, sound processing takes a lot of time. In order to improve the construction of user experience platform, C/S mode is used for development. System development language mainly: $\mathrm{C}++, \mathrm{C \#}$, Asp.net, HTML and html5 technology. C++ programming language features high operating efficiency and is very suitable for sound data processing. C\# language can write platform interface; Asp.net technology is used to develop the platform performance management and student management modules. HTML for web page beautification; Html5 is used for mobile APP development. The data transmission module is one of the core functions of the system. The platform USES multi-thread transmission, the system is deployed in the LAN, and the transmission capacity is improved by using FTP service transmission. The data store USES SQLServer2012 as the storage database. FIG. 4 shows the technical framework of the platform. The platform also adds data mining technology. Data mining technology mainly mines information stored in the database, including students' personal information, training information and performance information. Information mining can provide learning guidance for music learning and improve learning efficiency.

\section{Conclusion}

With the development of information technology, the disadvantages of music teaching can be solved by computer technology. Aiming at the problems existing in the teaching of vocal music platform, this paper USES computer technology to solve them and constructs a comprehensive platform for vocal music teaching. The platform proposes that data mining technology can be used to analyze students' learning status. Virtual training platform can provide online learning help for students, which solves the need of face-to-face guidance by teachers in vocal learning. With the continuous maturity of block chain technology, the application of it in vocal music teaching will improve the quality of vocal music teaching ${ }^{[7]}$.

\section{References}

[1] Liu Yuan. The vocal music teaching model for normal universities facing the new century [J]. Music,2007(3):190-191.

[2] Lu Yu. Preliminary exploration of computer-assisted vocal music teaching [J]. Art of music: journal of Shanghai music college,1999(1):66-70.

[3] Chen Hu, Zhao Kaidong. Application of modern information technology to optimize vocal music teaching [J]. Education research,2006(10):78-80.

[4] Zhao Yi. Research on vocal music teaching model based on constructivism theory [J]. Music creation,2012(3):187-189.

[5] Jiang Changqing. Study on "virtualization" mode construction of instrumental ensemble of college students from the perspective of computer music [J]. Music creation,2017(11):170-172.

[6] Li Bo, Chen Zhigang, huang rui, et al. Music recommendation algorithm based on LDA model [J]. Computer engineering,2016,42(6):175-179.

[7] Iin Yifu. Demand analysis and technical framework of blockchain + education [J]. Education China,2017(9):62-68. 